\title{
ISOMETRIES OF SPHERES
}

\author{
ULRICH EVERLING
}

(Communicated by Palle E. T. Jorgensen)

\begin{abstract}
In a Euclidean space of dimension two or more, any mapping that preserves unit distance is an isometry; this is the theorem of Beckmann and Quarles. We prove a similar theorem for spheres, assuming that a given distance less than a quarter great circle is preserved.
\end{abstract}

\section{INTRODUCTION}

Let $X$ be a Euclidean space of dimension 2 or more, $f: X \rightarrow X$ any mapping, and assume given an $s>0$ such that $f$ maps every two points of distance $s$ to points of distance $s$. Then $f$ is an isometry. This was first proved in [1]; other proofs were found later: [2], [3], [4], [8]. Similar theorems were proved by [5] and [6] for hyperbolic spaces, and by [9] for mappings of circles in an inversive plane. A survey of results, counter-examples and proofs is in [7].

For spheres, [6] treats the special case of $s=\pi / 3$, and [10] the case where $s=\pi / 2$ and $f$ is a homeomorphism. We drop the bijectivity assumption in the latter case and generalize the former to any $s<\pi / 2$.

Theorem. Let $E$ be a Euclidean space of dimension $n \geqq 3$ and $S \subset E$ its unit sphere with the geodesic distance $d(x, y)=\arccos \langle x, y\rangle$. Let $f: S \rightarrow S$ be any mapping and $P_{f}=\{s>0 \mid \forall x, y \in S: d(x, y)=s \Longrightarrow d(f(x), f(y))=s\}$.

A. If $P_{f} \cap(0, \pi / 2) \neq \varnothing$, then $f$ is an isometry.

B. If $f$ is continuous and $\pi / 2 \in P_{f}$, then $f$ is an isometry.

Remark. Without continuity, B would be false because $f(x)$ might be $\pm x$ with varying signs. The proof of $B$ is independent of [10], and answers a question of [11].

The author thanks Alexander Reznikov for a discussion on conceivable extensions of the Beckmann-Quarles theorem.

\section{Proof of STATEMENT A}

Summary of proof. By induction on $n$, we shall reduce to $n=3$. It will be sufficient to prove that inf $P_{f}=0$. Starting with one given $s \in P_{f}, s<\pi / 2$,

Received by the editors February 3, 1994.

1991 Mathematics Subject Classification. Primary 51F99, 51F20, 51N20, 51M04.

Key words and phrases. Isometry, sphere, projective plane, Beckmann-Quarles theorem. 
we shall construct a set of points whose distances are preserved by $f$, and find an $s^{\prime}<s$ among these distances, thereby approaching the goal of inf $P_{f}=0$. The case where $s$ is the edge length of an icosahedron needs an extra argument.

Throughout this proof, let $s \in P_{f} \cap(0, \pi / 2)$ be fixed, and

$$
h: \mathbb{R} \rightarrow[0,2 s], \quad h(t)=\arccos \left(\cos t \cdot \sin ^{2} s+\cos ^{2} s\right) .
$$

A spherical triangle of sides $s, s, s$ has interior angle $\varphi \in h^{-1}(s) \cap(\pi / 3, \pi / 2)$.

Reduction to $n=3$. Suppose the theorem is true for lower dimensions. Let $x, y \in S, d(x, y) \leq 2 s$. There is $z \in S$ such that $x, y \in S^{\prime}=\{p \in$ $S \mid d(p, z)=s\}$. There is an isometry $\alpha$ such that $\alpha(f(z))=z$, and $\alpha \circ f$ restricts to a mapping $g: S^{\prime} \rightarrow S^{\prime}$. The restriction of $d$ to $S^{\prime}$ is not the geodesic distance in $S^{\prime}$, but $S^{\prime}$ can be identified with the unit sphere of an $(n-1)$ dimensional space so that the distance is transformed by $h:[0, \pi] \stackrel{\sim}{\rightarrow}[0,2 s]$. Hence $\varphi \in P_{g}$ and by the induction hypothesis $g$ is an isometry. Therefore $d(\alpha f(x), \alpha f(y))=d(x, y)$, whence $(0,2 s] \subset P_{f}$ and $f$ is an isometry.

From now on we assume $n=3$.

Reduction to $\inf P_{f}=0$. Let $0<t<s$. There are points $x, y, z \in S$ such that $d(x, y)=t, d(y, z)=s-t$ and $d(x, z)=s$. For small $\varepsilon>0$, there are $k, l \in \mathbb{N}$ and $p_{0}, \ldots, p_{k+l} \in S$ such that $p_{0}=x, p_{k}=y, p_{k+l}=z$, $d\left(p_{i}, p_{i+1}\right)=\varepsilon, k \varepsilon<t+2 \varepsilon$ and $l \varepsilon<s-t+2 \varepsilon$. If $\varepsilon \in P_{f}$ it follows that $|d(f(x), f(y))-t|<2 \varepsilon$. So if $\inf P_{f}=0$, then $(0, s] \subset P_{f}$ and $f$ is an isometry.

It remains to prove that $\inf P_{f}=0$. Depending on the given value of $s$, we shall either conclude inf $P_{f}=0$ directly, or else find an $s^{\prime} \in P_{f}$ such that $s^{\prime} \leq h(\varphi / 2)<s$. In the latter case we start over with $s^{\prime}$ instead of $s$. Even if this were to repeat infinitely, the values of $s$ would converge to zero because $s \mapsto h(\varphi / 2)$ is continous.

The generic case. Let $p, q_{i} \in S$ such that

$$
d\left(p, q_{i}\right)=d\left(q_{i}, q_{i+1}\right)=s, q_{i+2} \neq q_{i} \quad(i=0,1,2, \ldots) .
$$

Then $d\left(q_{0}, q_{k}\right)=h(k \varphi)$. For the image points we have $d\left(f\left(q_{0}\right), f\left(q_{2}\right)\right) \in$ $\{0, h(2 \varphi)\}$. Since $q_{0}, q_{2}$ can be any points of distance $h(2 \varphi)$, we know

$$
\forall x, y \in S: d(x, y)=h(2 \varphi) \Longrightarrow d(f(x), f(y)) \in\{0, h(2 \varphi)\} .
$$

There are $x_{0}, \ldots, x_{m} \in S$ such that $d\left(x_{i}, x_{i+1}\right)=h(2 \varphi)$ and $d\left(x_{0}, x_{m}\right)=s$. Let $m$ be as small as possible $(m=2$ for small $s$, bigger $m$ for $s \approx \pi / 2)$. Then none of the $d\left(f\left(x_{i}\right), f\left(x_{i+1}\right)\right)$ can be zero. Hence $h(2 \varphi) \in P_{f}$. The conditions (1) are therefore preserved by $f$, whence

$$
\forall k \in \mathbb{N}: h(k \varphi) \in P_{f} \cup\{0\} .
$$

If $\varphi \notin \pi \mathbb{Q}$, this implies inf $P_{f}=0$.

If $\varphi=2 \pi a / b$ with $a, b \in \mathbb{N}$ coprime and $a>1$, then for some $k$ we have $k a \equiv 1(\bmod b)$ and

$$
P_{f} \ni s^{\prime}=h(k \varphi)=h(\varphi / a) \leq h(\varphi / 2)<s .
$$

The only remainig case is $\varphi=2 \pi / 5$. 
The icosahedral case. When $\varphi=2 \pi / 5$, the points of $V=\left\{ \pm p, \pm q_{0,1,2,3,4}\right\}$ are the vertices of an icosahedron. To construct some more points whose distances are preserved by $f$, the auxiliary set

$$
P_{\bar{f}}^{\leq}=\{t>0 \mid \forall x, y \in S: d(x, y) \leq t \Longrightarrow d(f(x), f(y)) \leq t\}
$$

will be used. It contains $2 s$ because

$$
d(x, y) \leq 2 s \Longleftrightarrow \exists z, d(x, z)=d(y, z)=s .
$$

The small star-shaped area

$$
\star=\{x \in S \mid \forall v \in V \backslash\{-p\}: d(v, x) \leq 2 s\}
$$

contains $p$ and has five corners at distance

$$
t=\frac{1}{10}(\sqrt{2 \sqrt{5}-1} \cdot(1+\sqrt{5})-3+3 \sqrt{5}) \approx 13^{\circ}
$$

from $p$, whereas $s=\arccos (1 / \sqrt{5}) \approx 63^{\circ}$. If $x, y \in S, d(x, y) \leq t$, then $p, q_{i}$ can be chosen so that $x=p$ and $y \in \star$. There is an isometry $\alpha$ such that $\alpha f(p)=p, \alpha f\left(q_{i}\right)=q_{i}$. Then $f(\star) \subseteq \star$, so $d(f(x), f(y)) \leq t$. This means $t \in P_{f}^{\leq}$, and since $P_{f}^{\leq}$is closed under addition, we have $3 t \in P_{f}^{\leq}$.

Now consider points $q, u, v, w$ such that

$$
\begin{array}{cc}
d(q, u)=d\left(q_{0}, u\right)=s, & d(q, v)=d\left(q_{1}, v\right)=s, \\
d(u,-p) \leq 2 s, & d(v,-p) \leq 2 s,
\end{array}
$$$$
d(p, q)=s, d\left(q_{0}, q\right) \leq 3 t, d\left(q_{1}, q\right) \leq 3 t,
$$

and $w \neq q$. The points $u, v, w$ are well defined depending on $q$. When $q$ runs through the arc segment defined by (2), the point $w$ runs through a curve segment where it meets the circle

$$
d\left(-q_{2}, w\right)=s
$$

exactly once. (With (2) and (4), $w \neq q$ is redundant.) The conditions (2)-(4) are all preserved by $f$. The distance $s^{\prime}=d\left(q, q_{1}\right) \approx 27^{\circ}<h(\varphi / 2)$ belongs to $P_{f}$, and the proof is finished.

\section{Proof of STATement B}

Summary of proof. Let $n=3$. Then $f$ may be assumed to fix the vertices of a spherical octahedron. The three great circles formed by the edges of the octahedron are mapped each into itself by $f$. Any point other than the vertices is characterized up to sign by the points of these great circles to which it is orthogonal. Between these three point pairs there is one relation. This relation must be preserved by the restrictions of $f$ to the three great circles, which is a strong requirement (the homomorphism property of $k$ below). From this it follows easily that $f$ is an isometry. The ambiguity of antipodal points is avoided by projection to the projective plane. Some care must be taken because points might unexpectedly be mapped to a vertex of the octahedron as $f$ is not assumed injective.

Reduction to $n=3$. The condition $d(x, y)=\pi / 2$ is now simply $x \perp y$. Hence the reduction is now simpler than for A because $\{p \in S \mid d(p, z)=s\}$ is itself the unit sphere of the Euclidean subspace $z^{\perp}$. 
Proof for $n=3$. Let $E=\mathbb{R}^{3}$ with the standard scalar product, and $\mathbb{P}^{2}$ the real projective plane. The latter inherits from $E$ the orthogonality relation $\perp$.

For any $x \in S$ there are $y, z \in S$ such that $x \perp y \perp z \perp x$. It follows that $f(-x)= \pm f(x)$. Hence $f$ induces a mapping $g: \mathbb{P}^{2} \rightarrow \mathbb{P}^{2}$ of the real projective plane, and $x, y \in \mathbb{P}^{2}, x \perp y \Longrightarrow g(x) \perp g(y)$. Also, $g$ is continuous. We may assume that $e_{0}=(1: 0: 0), e_{1}=(0: 1: 0), e_{2}=(0: 0: 1)$ are fixed points of $g$.

Let $j_{i}: \mathbb{P}^{1} \stackrel{\sim}{\rightarrow} e_{i}^{\perp} \hookrightarrow \mathbb{P}^{2}$,

$$
j_{0}(u: v)=(0: u: v), j_{1}(u: v)=(v: 0: u), j_{2}(u: v)=(u: v: 0) .
$$

Then there are continuous mappings

$$
h_{i}: \mathbb{P}^{1} \rightarrow \mathbb{P}^{1}, \quad g \circ j_{i}=j_{i} \circ h_{i} \quad(i=0,1,2) .
$$

Let $\varrho: \mathbb{P}^{1} \rightarrow \mathbb{P}^{1}, \varrho(u: v)=(-v: u)$. Then $h_{i} \circ \varrho=\varrho \circ h_{i}$.

The set $\mathbb{P}^{1} \cup\{(0: 0)\}$ has a multiplication $(a: b) \cdot(c: d)=(a c: b d)$, with identity element $1=(1: 1)$, and $G=\mathbb{P}^{1} \backslash\{(0: 1),(1: 0)\}$ is a group. For $(u, v) \in \mathbb{P}^{1}$ let $(u: v)^{\vee}=(v: u)$; in $G$ this is the inverse. We shall show that the $h_{i}$ are slight modifications of a group endomorphism of $G$.

Let $q_{0}, q_{1}, q_{2} \in \mathbb{P}^{1}$. Then by the determinant criterion,

$$
\bigcap_{i}\left(j_{i} \varrho q_{i}\right)^{\perp} \neq \varnothing \Longleftrightarrow q_{0} \cdot q_{1} \cdot q_{2} \in\{1,(0: 0)\} .
$$

In that case it follows that $h_{0}\left(q_{0}\right) \cdot h_{1}\left(q_{1}\right) \cdot h_{2}\left(q_{2}\right) \in\{1,(0: 0)\}$; and if two of the $h_{i}\left(q_{i}\right)$ are in $G$, then the product is 1 .

Since $h_{i}$ are continuous and $h_{i}(0: 1)=(0: 1)$ and $h_{i}(1: 0)=(1: 0)$, there are $p_{1}, p_{2} \in G$ such that $h_{1}\left(p_{1}\right), h_{2}\left(p_{2}\right) \in G$. Then $p_{0}:=p_{1}^{\vee} \cdot p_{2}^{\vee} \in G$ and $h_{0}\left(p_{0}\right) \cdot h_{1}\left(p_{1}\right) \cdot h_{2}\left(p_{2}\right)=1$. Let $k_{i}(q)=h_{i}\left(p_{i}\right)^{\vee} \cdot h_{i}\left(p_{i} \cdot q\right)$. Then $k_{i}(1)=1$ and

$$
q_{0} \cdot q_{1} \cdot q_{2}=1 \Longrightarrow k_{0}\left(q_{0}\right) \cdot k_{1}\left(q_{1}\right) \cdot k_{2}\left(q_{2}\right) \in\{1,(0: 0)\} \text {. }
$$

Let $q \in \mathbb{P}^{1}$ and $k_{0}(q) \in G$. Then $k_{0}(q) \cdot k_{1}\left(q^{\vee}\right) \cdot k_{2}(1)=1$, whence $k_{1}\left(q^{\vee}\right)=$ $k_{0}(q)^{\vee}$. Likewise, $k_{i}\left(q^{\vee}\right)=k_{j}(q)^{\vee}$ for $i \neq j$. Hence

$$
H:=k_{0}^{-1}(G)=k_{1}^{-1}(G)=k_{2}^{-1}(G),
$$

$\forall q \in H: \quad k(q):=k_{0}(q)=k_{1}(q)=k_{2}(q), \quad q^{\vee} \in H, \quad k\left(q^{\vee}\right)=k(q)^{\vee}$.

If $q, r \in H$, then $k(q) k(r) k\left((q r)^{\vee}\right)=1$. So $H$ is a subgroup of $G$ and $k: H \rightarrow G$ is a homomorphism.

Also, $k_{0}(-1: 1)=k_{0}\left(p_{0}^{\vee} \cdot \varrho\left(p_{0}\right)\right)=h_{0}\left(p_{0}\right)^{\vee} \cdot \varrho\left(h_{0}\left(p_{0}\right)\right) \in G$, so $H$ meets both connected components of $G$. On the other hand, $H$ is open, so $H=G$. Let $\psi: \mathbb{R} \rightarrow \mathbb{R},(\psi(t): 1)=k(t: 1)$. Then $\varepsilon=\psi(-1) \in\{ \pm 1\}$.

For $q \in \mathbb{P}^{2}$ we have $h_{i}(q)=h_{i}\left(p_{i}\right) k\left(p_{i}^{\vee} \cdot q\right)$ and therefore $h_{i}(p \cdot q)=k(p)$. $h_{i}(q)$ for $p \in G$. In particular let $h_{i}(1)=\left(c_{i}: 1\right)$. Then $h_{i}(-1: 1)=\left(\varepsilon c_{i}: 1\right)$, so $\left(c_{i}: 1\right) \perp\left(\varepsilon c_{i}: 1\right)$, which means $\varepsilon=-1$ and $c_{i}^{2}=1$. After suitable changes of signs of $g$ we may assume $c_{i}=1$, and then all $h_{i}=k$.

For $p=(x: y: z) \in \mathbb{P}^{2} \backslash\left\{e_{0}, e_{1}, e_{2}\right\}$, let $q_{0}=(y: z), q_{1}=(z: x), q_{2}=$ $(x: y)$. Then $p \in \cap\left(j_{i} \varrho q_{i}\right)^{\perp}$. Hence

$$
g(p) \in \bigcap_{i}\left(g j_{i} \varrho q_{i}\right)^{\perp}=\bigcap_{i}\left(j_{i} \varrho k q_{i}\right)^{\perp}=\{(\psi(x): \psi(y): \psi(z))\} .
$$


In particular $g(1: 1: 1)=(1: 1: 1)$. Let $s, t \in \mathbb{R}^{*}, s+t \neq 0$. Then

$$
\begin{aligned}
(s: t:-s-t) \perp(1: 1: 1) & \Longrightarrow(\psi(s): \psi(t): \psi(-s-t)) \perp(1: 1: 1) \\
& \Longrightarrow \psi(-s-t)=-\psi(s)-\psi(t) .
\end{aligned}
$$

Hence $\psi$ is an endomorphism of the field $\mathbb{R}$. This field has no endomorphisms but the identity. By (5), $g$ itself is the identity. This means $f(x)= \pm x$, and the sign is constant because $f$ is continuous. So $f$ is in fact an isometry.

\section{REFERENCES}

1. F. S. Beckmann and D. A. Quarles, Jr., On isometries of Euclidean spaces, Proc. Amer. Math. Soc. 4 (1953), 810-815.

2. Walter Benz, An elementary proof of the theorem of Beckmann and Quarles, Elem. Math. 42 (1987), 4-9.

3. Richard L. Bishop, Characterizing motions by unit distance invariance, Math. Mag. 46 (1973), 148-151.

4. Krzysztof Ciesielski, The isometry problem, Math. Intelligencer 10 (1988), 44.

5. Bijan Farrahi, A characterisation of isometries of absolute planes, Resultate Math. 4 (1981), 34-48.

6. A. V. Kuz'minyh, Mappings that preserve unit distance, Siberian Math. J. 20 (1979), 417-421.

7. Hanfried Lenz, Bemerkungen zum Beckmann-Quarles-Problem, Mitt. Math. Ges. Hamburg 12 (1991), 429-446.

8. J. A. Lester, Transformatins of $n$-space which preserve a fixed square-distance, Canad. J. Math. 31 (1979), 392-395.

9. _ A Beckmann-Quarles type theorem for Coxeter's inversive distance, Canad. Math. Bull. 34 (1991), 492-498.

10. Bogdan Mielnik and Themistocles M. Rassias, On the Aleksandrov problem of conservative distances, Proc. Amer. Math. Soc. 116 (1992), 1115-1118.

11. Th. M. Rassias, Mappings that preserve unit distance, Indian J. Math. 32 (1990), 275-278.

Mathematisch-Geographische FakUltät, Katholische Universität Eichstätt, OstenSTRASSE 26-28, 85071 EICHSTÄTT, GERMANY

E-mail address: ulrich.everling@ku-eichstaett.de 